INRA Prod. Anim., 2003, 16 (4), 275-278
J.B. COULON ${ }^{1}$, E. ROCK ${ }^{2}, Y . N O \ddot{E} L^{3}$

${ }^{1}$ INRA, Unité de Recherches sur les Herbivores, Theix, 63122 Saint-Genès Champanelle

${ }^{2}$ INRA, Unité Maladies Métaboliques et Micronutriments, Theix, 63122 Saint-Genès Champanelle

${ }^{3}$ INRA, Unité de Recherches en

Technologie et Analyses Laitières, BP 89, 39801 Poligny cedex

Courriel:jbc@clermont.inra.fr

\title{
Caractéristiques nutritionnelles des produits laitiers et variations selon leur origine
}

$\mathrm{Au}$ cours des dernières années de nombreux travaux ont été réalisés sur les caractéristiques sensorielles des fromages d'AOC, dans le cadre général de l'objectivation de leur liaison à leur terroir (voir Martin et al 2003, dans ce même dossier). De plus en plus, et au-delà des produits AOC, les consommateurs sont à la recherche d'information sur les caractéristiques nutritionnelles des aliments qu'ils consomment, et des produits laitiers en particulier. Les fromages d'AOC sont particulièrement concernés par cette question, dans la mesure où les conditions de leur production peuvent conduire à des caractéristiques nutritionnelles spécifiques. Celles-ci relèvent schématiquement de deux domaines distincts, d'une part les caractéristiques liées à la microbiologie des laits et des fromages et d'autre part celles liées à leur teneur en macro ou micro-constituants d'intérêt nutritionnel (protéines, lipides, vitamines, minéraux...).

L'objectif de ce texte est de fournir quelques points de repères sur le rôle des micronutriments et de la microflore sur la santé, sur les liens entre la microflore digestive de l'Homme et son système immunitaire, et sur les actions déjà engagées ou en projet pour identifier et comprendre le rôle de la consommation de fromages au lait cru sur la santé humaine.

\section{1 / Les micronutriments des produits laitiers}

Il est important de considérer que tout aliment interagit avec l'organisme et donc de le placer dans un contexte santé, sans pour autant déprécier les conséquences de cette interaction sur les sens et donc sur les plaisirs à le consommer.

Dans ce contexte, le rôle des aliments sur la santé est relativement bien connu en raison des conséquences visibles et à court terme d'une dénutrition. Les aliments apportent tout ce qui est essentiel pour la croissance, le maintien et le fonctionnement des structures de l'organisme (muscle, os ...) et donc de la santé générale. Cette action est principalement due à l'apport d'énergie contenue dans les aliments sous forme de protéines, lipides et glucides. Une analyse plus fine montre que les aliments contiennent des éléments non énergétiques parmi lesquels on trouve des micronutriments (minéraux, oligo-éléments, vitamines, caroténoïdes et polyphénols). Des carences sévères en ces éléments se traduisent rapidement par des pathologies évidentes, et des apports insuffisants en micronutriments pourraient être à l'origine de pathologies apparaissant avec l'âge. Ces maladies recouvrent essentiellement les maladies cardiovasculaires et certains cancers, mais aussi les maladies affectant l'os (ostéoporose) ou les yeux (cataracte, dégénérescence maculaire liée à l'âge). Si des études épidémiologiques ont montré une telle implication, il reste encore à démontrer une relation de cause à effet de ces micronutriments : ceci fait l'objet des recherches actuellement menées sur les actions de ces micronutriments globalement sur l'organisme, mais aussi à l'échelle des cellules et des molécules qui le composent.

Les produits laitiers sont reconnus comme une source importante de protéines digestibles, de vitamine A et de calcium. Le calcium des produits laitiers présente une bonne biodisponibilité et, associé au phosphore, intervient directement dans la constitution de la masse osseuse et dans la protection contre la fragilisation des os à l'origine de l'ostéoporose. Les produits laitiers sont également des sources de minéraux et oligo-éléments, mais aussi de vitamine $\mathrm{K}$ et, pour les produits fermentés, de vitamines B (Debry 2001, Martin 
2001). Ainsi, les laitages sont considérés comme source alimentaire pour le fer à $6 \%$, le cuivre, le zinc et le magnésium à $15-20 \%$, le phosphore à $39 \%$ et le calcium à $67 \%$. Ces données sur les minéraux sont d'autant plus importantes à considérer que les données épidémiologiques montrent qu'un pourcentage non négligeable de la population française a une consommation inférieure aux apports nutritionnels conseillés (Volatier et al 2000). Pour les caroténoïdes et les polyphénols, les données sont plus éparses et des travaux initiés à l'INRA de Theix apporteront quelques précisions. Les effets santé des micronutriments des produits laitiers sont essentiellement connus pour le calcium et le phosphore (Gueguen 2002). Cependant, les autres minéraux et phyto-micronutriments devront être pris en considération en raison notamment de leur action potentielle pour la lutte contre le stress oxydant. Les produits laitiers contiennent aussi des lipides parmi lesquels certains pourraient avoir un effet santé intéressant, comme les acides linoléiques conjugués ou CLA. A côté des nutriments présentant des effets favorables, il en existe certains pouvant avoir un rôle néfaste comme des contaminants (pesticides, métaux lourds) et d'autres comme les acides gras saturés ou les acides gras trans, supposés être à l'origine des maladies cardiovasculaires, comme le montrent certaines études épidémiologiques.

\section{2 / Microflore des produits laitiers et propriétés microbiologiques}

Les liens entre les propriétés microbiologiques des laits crus et des fromages fabriqués à partir de ces laits, et les conditions de production des laits font l'objet d'études en cours. Les fromages, en tant qu'aliment pour l'Homme, contiennent une microflore importante et diversifiée, dont le rôle sur la santé humaine a été mis en question récemment, à l'initiative des professionnels.

La microflore digestive de l'Homme est abondante (Moreau 2001a) : $10^{14}$ bactéries localisées essentiellement dans le côlon, soit environ $10^{11}$ bactéries/g de fèces. Elle est caractérisée par un équilibre entre différentes espèces de bactéries, avec une microflore dominante (supérieure à $10^{6}$ bactéries $/ \mathrm{g}$ contenu), et des microorganismes en transit $\left(10^{8}\right.$ à $10^{9}$ g contenu). Ces microorganismes en transit proviennent soit de l'alimentation (laits fermentés, fromages...), soit de prescriptions médicamenteuses (levures). A titre d'exemple, un yaourt représente l'ingestion de plus de $10^{9}$ bactéries.

L'intestin est un organe immunitaire essentiel puisque la muqueuse intestinale de l'intestin grêle et du côlon contient 60 à $70 \%$ des cellules immunes de l'Homme (Moreau 2001b). Ce système, appelé 'système immunitaire de l'intestin' (SII), a deux fonctions : (1) assurer la synthèse d'anticorps, en particulier les immunoglobulines A (IgA), pour protéger l'organisme contre les microorganismes opportunistes entéropathogènes, (2) assurer la tolérance orale, en supprimant le développement de réponses immunes contre les protéines alimentaires et bactériennes étrangères à l’organisme.

Le rôle de la microflore intestinale résidente sur la modulation de ces fonctions est encore insuffisamment connu, bien que des arguments expérimentaux et cliniques le soutiennent. Son importance a conduit à s'interroger sur les effets immunomodulateurs de l'ingestion de 'probiotiques' (microorganismes vivants qui, lorsqu'ils sont ingérés en quantité adéquate, produisent un bénéfice pour la santé de l'Homme). L'aliment 'prébiotique' contient quant à lui des molécules fermentescibles susceptibles d'agir favorablement sur les bactéries intestinales et sur les probiotiques (Rebecchi et Bottazi 2001). La microflore digestive joue un rôle crucial dans l'établissement du SII chez le jeune (Moreau 2001b), démontré par des travaux sur modèle animal et par des observations chez les bébés. Les effets de l'ingestion de probiotiques ont été et sont explorés chez l'Homme : la démonstration d'une persistance durable n'est pas faite (durée de survie connue : 2 à 20 jours ; taux de survie : $30 \%$ ) ; la dose ingérée doit conduire à une concentration supérieure à 107 bactéries/g fèces ; les effets sont différents selon les espèces bactériennes, l'effet immunostimulant est lié à la souche; le rôle des métabolites est encore questionné. Les données actuelles ne concernent que les microorganismes présents dans les laits fermentés (bactéries lactiques) ou utilisés comme médicaments (levures).

Alors que les laits fermentés contiennent un nombre très limité de souches bactériennes (1 à 3), la richesse et la diversité de la microflore des fromages, notamment ceux fabriqués au lait cru, seraient-elles un atout ? La consommation de fromages est une pratique alimentaire courante. Il est donc intéressant de s'interroger sur les effets éventuels de cette consommation sur l'équilibre de la microflore intestinale de l'Homme et sur le système immunitaire (Moreau et Vuitton 2002). L'Homme sain a par définition une microflore intestinale en bon équilibre capable de remplir les fonctions immunomodulatrices nécessaires. Mais cet équilibre peut être perturbé par de nombreux stress (par exemple, gastro-entérites, rhumes, fatigue ... ou prise d'antibiotiques). La consommation régulière de fromages pourrait-elle compenser naturellement ces déséquilibres, contribuant à préserver le bien-être et la santé de l'Homme et à prévenir à long terme certaines pathologies ? Les effets probiotiques de différentes souches de bactéries lactiques (streptocoques, lactobacilles et bifidobactéries), de propionibactéries et de levures ont été étudiés et des propriétés immunomodulatrices ont été mises en évidence pour certaines souches. Des effets protecteurs chez l'Homme (nourrisson, adulte) ont été observés (diarrhées infectieuses, hypersensibilités alimentaires, maladies inflammatoires du tube digestif ...) sans que les mécanismes soient encore élucidés. Les revues récentes de Mogensen et al (2002a) et Ouwehand et al (2003) ne traitent pas des fro- 
mages, mais fournissent cependant un intéressant état des connaissances sur les effets bénéfiques pour la santé humaine des bactéries lactiques, utilisées principalement dans des préparations alimentaires probiotiques. Un inventaire des microorganismes alimentaires avec l'histoire documentée de leur usage a été établi à l'initiative de la Fédération Internationale de Laiterie et de European Food and Feed Cultures Association (Mogensen et al 2002b).

\section{3 / Variations des micronutri- ments selon l'origine des produits laitiers}

Plusieurs travaux récents ont étudié les relations entre la nature de l'alimentation offerte et la teneur en micronutriments d'intérêt pour la santé humaine. En particulier, nous avons comparé, en conditions expérimentales sur des laits individuels, l'effet de grands types de rations de base (Martin et al 2002). Six rations couvrant une large gamme de situations alimentaires (rations « riche en concentrés » (65\%), « ensilage de maïs », « ensilage de ray-grass », " foin de ray-grass », « foin de prairie naturelle », et « herbe de prairie naturelle pâturée ») ont été offertes pendant six semaines à des lots de huit vaches laitières (quatre de race Tarentaise et quatre de race Montbéliarde) en phase descendante de lactation. A l'issue de la période expérimentale, les teneurs des laits en calcium, en phosphore, en caroténoïdes, en vitamines $\mathrm{A}$ et $\mathrm{E}$, en acides gras majeurs et mineurs (dont les isomères des C18:1 et des CLA (acide linoléique conjugué)), ainsi que le potentiel antioxydant ont été mesurés sur des laits individuels. Les teneurs en polyphénols et en composés à activité antimicrobienne (lactoferrine, thiocyanate) ont été dosées sur les laits de mélange des différents lots.

Les régimes «riche en concentré » ou « ensilage de maïs » s'opposent aux régimes à base d'herbe, en particulier sous forme pâturée (figure 1). Les rations riches en concentré et en ensilage de maïs conduisent à des lait plus riches en phosphore et moins riches en beta-carotène et en vitamine $\mathrm{E}$ par rapport aux autres. Le statut antioxydant de ces laits est inférieur à celui mesuré sur des laits obtenus avec un régime à base d'herbe pâturée. L'herbe pâturée a également été à l'origine des laits les plus riches en acide oléique et en isomère cis9, trans 11 du CLA (acide ruménique) par rapport aux rations riches en concentré ou à base d'ensilage de maïs. Toutefois, l'effet du pâturage semble variable selon le stade de l'herbe : l'herbe pâturée à un stade précoce a conduit à des laits dont la teneur en acide ruménique a été deux fois plus élevée comparativement à une herbe pâturée à un stade tardif. Les premiers dosages de polyphénols, de lactoferrine et de thiocyanate semblent également révéler une variabilité selon la nature des fourrages distribués aux animaux : les laits issus du pâturage ont été les plus riches en composés phénoliques et les laits issus des régimes à base d'herbe (quels que soient sa nature et son mode de conservation) ont été plus riches en thiocyanate comparativement aux laits issus des régimes à base de concentré ou d'ensilage de maïs. En revanche, les laits issus du régime à base de concentré ont été les plus riches en lactoferrine.

Par ailleurs, dans un essai récent en cours de dépouillement, nous avons pu vérifier que lors de la transformation du lait en fromage,

Figure 1. Liaison entre la nature de la ration et la teneur en micronutriments des laits. (d'après Martin et al 2002).

Les acides gras du lait sont présentés avec les abréviations suivantes : C4 : acide butyrique, C6 : acide caproïque, C10 : acide caprique, C14 : acide myristique, C18:0 : acide stéarique, C18:1 : acide oléique, CLA : acides linoléiques conjugués.

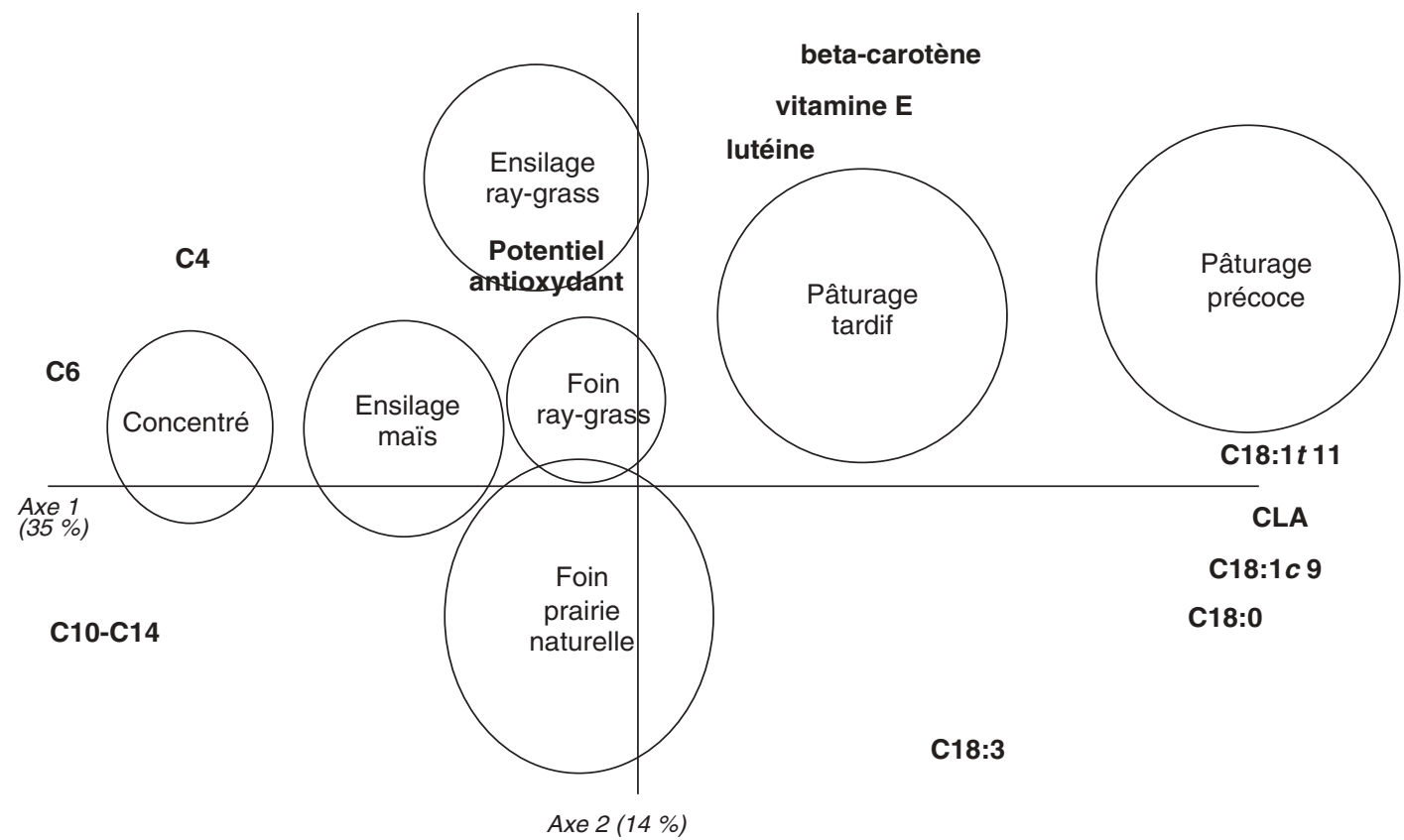


les carotènes, la vitamine $\mathrm{E}$ et les acides gras ne subissent que très peu de modifications, quel que soit le type de fromage (Cantal ou SaintNectaire) et que le lait soit cru ou pasteurisé.

Ces résultats confirment et précisent les données obtenues par ailleurs sur l'effet des régimes à base d'herbe sur la lutéine (Astier et $a l$, non publié), le beta-carotène (Coulon et Priolo 2002, Prache et al 2002), les composés phénoliques (Poulet et al 2002), les acides gras (Collomb et al 1999, Chilliard et al 2001) ou le pouvoir antioxydant des produits laitiers (Pizzoferrato et al 2000).

\section{Conclusions}

Si les grandes familles de micronutriments pouvant avoir un intérêt pour la santé humaine sont maintenant de mieux en mieux connues (cf l'ouvrage de synthèse sur ce sujet de Debry, 2001), la teneur des produits laitiers en ces micronutriments et leurs facteurs de variation sont encore très peu documentés. Les quelques résultats disponibles, obtenus en conditions expérimentales, sont cependant prometteurs : ils montrent que la teneur du lait en certains de ces micronutriments peut varier sensiblement en fonction de la nature de l'alimentation offerte aux animaux. Ils doivent être confirmés et validés sur des fro- mages, et sur une large gamme de systèmes de production et de technologies fromagères ${ }^{(1)}$ Outre ces données sur la composition des produits laitiers, il sera nécessaire dans l'avenir de préciser le rôle de ces micronutriments dans la nutrition préventive au travers d'études permettant de déterminer leur biodisponibilité. Enfin, il serait utile de déterminer, dans le cadre des consommations actuelles des produits laitiers, leur contribution dans l'alimentation humaine et leur place dans la nutrition préventive.

En ce qui concerne le volet microbiologique, si les connaissances scientifiques relatives aux microorganismes des laits fermentés se sont beaucoup développées, il reste beaucoup à faire avec les fromages. Une stratégie d'investigation des effets immunomodulateurs éventuels de la consommation de fromages au lait cru a été élaborée par l'INRA, la Faculté de médecine et de pharmacie de Besançon, sur modèle animal et sur modèle clinique (Moreau et Vuitton 2002). Le programme de recherche sur modèle clinique a démarré fin 2002 avec un financement national de recherche hospitalière. Il vise à mesurer des indicateurs du fonctionnement du système immunitaire sur des volontaires stressés par une prise d'antibiotiques et les effets sur ces indicateurs de la consommation de fromages expérimentaux dans des conditions contrôlées.

\section{Références}

Chilliard Y., Ferlay A., Doreau M., 2001. Effect of different types of forages, animal fat or marine oils in cow's diet on milk fat secretion and composition, especially conjugated linoleic acid (CLA) and polyunsaturated fatty acids. Livest. Prod. Sci., 70, 31-48.

Collomb M., Bütikofer U., Spahni M., Jeangros B., Bosset J.O., 1999. Composition en acides gras et en glycérides de la matière grasse du lait de vache en zones de montagne et de plaine. Sci. Alim., 19, 97-110.

Coulon J.B., Priolo A., 2002. La qualité sensorielle des produits laitiers et de la viande dépend des fourrages consommés par les animaux. INRA Prod. Anim., 15, 333-342.

Debry G., 2001. Lait, nutrition et santé. Editions Tec et Doc, Lavoisier, 566 p.

Gueguen L., 2002. Quoi de neuf sur le calcium et la santé ? NAFAS Science, 7, 13-20.

Martin A., 2001. Apports Nutritionnels Conseillés pour la population Française. Editions Tec et Doc, Lavoisier, Paris.

Martin B., Ferlay A., Pradel P., Rock E., Grolier P., Dupont D., Gruffat D., Besle J.M., Ballot N., Chilliard Y. Coulon J.B., 2002. Variabilité de la teneur des laits en constituants d'intérêt nutritionnel selon la nature des fourrages consommés par les vaches laitières. Renc. Rech. Rum., 9, 347-350.

Martin B., Buchin S., Hurtaud C., 2003. Conditions de production du lait et qualités sensorielles des fromages. INRA Prod. Anim., 16, 283-288.

Mogensen G., Salminen S., O'Brien J., Ouwehand A. Hozapfel W., Shortt C., Fondén R., Miller G.D., Donohue D. Playne M. Crittenden R., Bianchi Salvadori B., Zink R. 2002a. Food Microorganisms - Health benefits, safety evaluation and strains with documented history of use in foods. Bulletin of the IDF, 377, 4-9.

Mogensen G., Salminen S., O'Brien J., Ouwehand A., Hozapfel W., Shortt C., Fondén R., Miller G.D., Donohue D., Playne M., Crittenden R., Bianchi Salvadori B., Zink R. $2002 \mathrm{~b}$. Inventory of microorganisms with a documented history of use in food. Bulletin of the IDF, 377, 10-19.
Moreau M.C., 2001a. Les probiotiques : des microoganismes bénéfiques pour notre système immunitaire? CholéDoc, $\mathrm{n}^{\circ}$ 63, Janvier/février. À consulter en ligne sur le site du cerin (http://www.cerin.org/periodiques/choledoc/choledoc_63.asp)

Moreau M.C., 2001b. Microflore intestinale, prébiotiques, probiotiques et immunomodulation. NAFAS Science, 6, 19-26.

Moreau M.C., Vuitton D.A., 2002. Le fromage et les bénéfices du vivant en matière de santé : améliorations des défenses immunitaires. Congrilait, Cd-rom

Ouwehand A.C., Salvadori B.B., Fondén R., Mogensen G., Salminen S., Sellars R., 2003. Health effects of probiotics and culture-containing dairy products in humans Bulletin of the IDF, 380, 4-19.

Pizzoferrato L, Manzi P, Rubino R, Fedele V., Pizzillo M., 2000. Degree of antioxidant protection in goat milk and cheese: the effect of feeding systems. Proc 7th Intl Conf. on goats, 2, 580-583.

Poulet J.L., Fraisse D., Viala D., Carnat A., Pradel P., Martin B., Lamaison J.L., Besle J.M., 2002. Flavonoids in forages: compositon and possible effects on milk quality. In : J.L. Durand, J.C. Emile, C. Huyghe and G. Lemaire (eds), Multi-fonction grasslands: quality forages, animal products and landscapes, British Grassland Society, 590-591.

Prache S., Priolo A., Tournadre H., Jailler R., Dubroeucq H., Micol D, Martin B, 2002. Traceability of grass-feeding by quantifying the signature of carotenoid pigments in herbivores meat, milk and cheese. In : J.L. Durand, J.C. Emile, C. Huyghe and G. Lemaire (eds), Multi-fonction grasslands: quality forages, animal products and landscapes, British Grassland Society, 592-593.

Rebecchi R., Bottazzi V., 2001. Bactéries lactiques pro-biotiques et nouveaux produits fonctionnels. NAFAS Science, 6, 27-38.

Volatier J.L., Maffre J., Couvreur A., et al, 2000. Enquête individuelle et nationale sur les consommations alimentaires (INCA). Tec \& Doc Lavoisier, Paris.

(1) Un programme vient de démarrer dans ce sens (Relation entre les conditions de production du lait et les teneurs en micronutriments d'intérêt pour la santé humaine dans le fromage : étude sur 4 types de fromage des Alpes du Nord et du Massif Central), dans le cadre conjoint du Pôle fromager Massif Central et du GIS Alpes du Nord. 